\title{
The Value of Intraoperative Parathyroid Hormone Monitoring in Localized Primary Hyperparathyroidism: A Cost Analysis
}

\author{
Lilah F. Morris, MD ${ }^{1}$, Kyle Zanocco, MD $^{2}$, Philip H. G. Ituarte, PhD $^{1}$, Kevin Ro ${ }^{1}$, Quan-Yang Duh, MD, FACS ${ }^{3}$, \\ Cord Sturgeon, MD, FACS ${ }^{2}$, and Michael W. Yeh, MD, FACS ${ }^{1}$ \\ ${ }^{1}$ Division of General Surgery, Endocrine Surgical Unit, David Geffen School of Medicine at UCLA, Los Angeles, CA; \\ ${ }^{2}$ Section of Endocrine Surgery, Department of Surgery, Northwestern University Feinberg School of Medicine, Chicago, \\ IL; ${ }^{3}$ Department of Surgery, University of California San Francisco, San Francisco, CA
}

\begin{abstract}
Background. Minimally invasive parathyroidectomy (MIP) is the preferred approach to primary hyperparathyroidism (PHPT) when a single adenoma can be localized preoperatively. The added value of intraoperative parathyroid hormone (IOPTH) monitoring remains debated because its ability to prevent failed parathyroidectomy due to unrecognized multiple gland disease (MGD) must be balanced against assay-related costs. We used a decision tree and cost analysis model to examine IOPTH monitoring in localized PHPT.
\end{abstract}

Methods. Literature review identified 17 studies involving 4,280 unique patients, permitting estimation of base case costs and probabilities. Sensitivity analyses were performed to evaluate the uncertainty of the assumptions associated with IOPTH monitoring and surgical outcomes. IOPTH cost, MGD rate, and reoperation cost were varied to evaluate potential cost savings from IOPTH.

Results. The base case assumption was that in welllocalized PHPT, IOPTH monitoring would increase the success rate of MIP from 96.3 to $98.8 \%$. The cost of IOPTH varied with operating room time used. IOPTH reduced overall treatment costs only when total assayrelated costs fell below $\$ 110$ per case. Inaccurate localization and high reoperation cost both independently increased the value of IOPTH monitoring. The IOPTH strategy was cost saving when the rate of unrecognized

(C) The Author(s) 2009. This article is published with open access at Springerlink.com

First Received: 21 May 2009;

Published Online: 3 November 2009

L. F. Morris, MD

e-mail: 1morris@mednet.ucla.edu
MGD exceeded $6 \%$ or if the cost of reoperation exceeded $\$ 12,000$ (compared with initial MIP cost of \$3733). Setting the positive predictive value of IOPTH at $100 \%$ and reducing the false-negative rate to $0 \%$ did not substantially alter these findings.

Conclusions. Institution-specific factors influence the value of IOPTH. In this model, IOPTH increased the cure rate marginally while incurring approximately $4 \%$ additional cost.

Minimally invasive parathyroidectomy (MIP), also known as focused parathyroidectomy or limited parathyroid exploration, is known to yield long-term cure rates equivalent to those achieved with conventional bilateral neck exploration. ${ }^{1,2}$ MIP requires relatively accurate preoperative localization, however. In most expert centers, MIP is now the preferred surgical approach to sporadic primary hyperparathyroidism (PHPT) when a single adenoma can be localized preoperatively. ${ }^{3}$ The fraction of patients who undergo MIP has increased over time and varies across institutions, ranging from 57 to $92 \%$ currently. ${ }^{4}$ This figure largely hinges on the accuracy of preoperative localization studies, most commonly technetium $99 \mathrm{~m}$ sestamibi scanning and ultrasound.

Many centers use intraoperative parathyroid hormone (IOPTH) monitoring as an adjunct to MIP. Although some experts consider it essential for success, others have questioned the added value that IOPTH monitoring brings when disease is adequately preoperatively localized. ${ }^{5}$ Drawbacks associated with IOPTH use include the cost of the assay, operating room (OR) time associated with waiting for results, and the potential for misleading the surgeon into performing unnecessary further exploration (false negatives). ${ }^{6}$ Published single-institution reports show a 
slight, statistically nonsignificant trend toward higher success rates for initial MIP when IOPTH monitoring is used: 95-97.5\% without IOPTH vs. 97-99\% with IOPTH. ${ }^{7-11}$ For patients with positive localization studies, the purpose of IOPTH monitoring is to unmask cases of multiple gland parathyroid disease (MGD) not recognized on imaging. In cases where the IOPTH decreases immediately after singlegland resection, the need to examine the other normal glands is obviated.

The addition of IOPTH monitoring to MIP increases the cost of a focused exploration. Whether this cost is justified by the potential prevention of failed operations remains debated. In addressing this topic, several questions must be considered: (1) What is the added cost of IOPTH monitoring? (2) What is the rate of unrecognized MGD in patients with positive localization? (3) What are the performance characteristics of IOPTH monitoring? (4) What is the cost of reoperation after initial surgery fails?

In this study, we use a decision tree and cost analysis to examine the influence of the aforementioned factors on the cost of IOPTH monitoring in localized PHPT.

\section{METHODS}

\section{Case Definition}

We created a decision-tree model to analyze the cost of IOPTH on the basis of the accuracy of preoperative localization studies, the cost of reoperation, MGD rate, and cost of IOPTH. A reference case scenario was created on the basis of a hypothetical 60-year-old woman with biochemically confirmed asymptomatic PHPT and no prior neck operations, who met the 2002 consensus criteria for parathyroidectomy. ${ }^{12}$ A literature review was conducted to obtain estimates of the costs and probabilities used in the model. We identified 17 studies involving 4,280 unique patients. Sensitivity analyses were performed to evaluate the uncertainty of the assumptions associated with IOPTH monitoring and surgical outcomes.

\section{Decision Model}

Decision analysis software (TreeAge Pro 2008; TreeAge Software, Williamstown, MA) was used to construct a decision model for the treatment of the reference case. The complete decision tree is shown in Fig. 1. Different treatment pathways were created for the two alternatives: first, MIP without the use of IOPTH, and second, MIP with the use of IOPTH. The selected probabilities are shown in Table 1. The hypothetical patient was assumed to be a surgical candidate with no history of neck surgery and that parathyroidectomy could be safely performed through a cervical incision (i.e., no sternotomy, thoracotomy, or thoracoscopy required for ectopic glands). The cure rate for initial parathyroidectomy without IOPTH was assigned a probability of $96.3 \%$. Relevant long-term complications of surgery included permanent recurrent laryngeal nerve damage and hypoparathyroidism. The risk of long-term complications resulting from initial parathyroid surgery was set at $1-2 \%$. The risks of temporary recurrent laryngeal nerve damage or hypoparathyroidism were not factored into the model. Failed initial parathyroidectomy led to one reoperation. Costs related to medical management were not considered given our assumption of an asymptomatic patient.

As shown in Fig. 1, we analyzed decision pathways where IOPTH is or is not used. We used the operating characteristics of IOPTH to determine the added value of IOPTH monitoring in patients with adequate preoperative localization of parathyroid adenomas. Studies analyzed generally used the Miami criterion to predict cure versus the need for continued exploration (IOPTH decrease $\geq 50 \%$ from the highest of either preincision or preexcision level at 10 min after gland excision). ${ }^{13}$ The costs and probabilities were based on distributions of values determined from reviewing the literature (Table 1 ).

\section{Costs}

Direct medical costs of surgery, complications, and IOPTH were estimated by using reported Medicare charge and reimbursement data, and are reported in Table 2 in 2005 U.S. dollars. We used a third-party payer perspective (Medicare diagnosis-related groups) to calculate costs (Table 2). Costs that were outside the health care system (e.g., transportation and lost-productivity costs) were not included in the analysis. Only costs that differed among the two treatment strategies were included. We calculated the cost of OR time via the following formula, derived from Medicare reimbursement for anesthesia: cost $=[6+($ time in minutes/15) * 17.78] (Table 3).

\section{Sensitivity Analysis}

The reference case scenario was tested by using sensitivity analysis to identify the uncertainty of the results. ${ }^{14}$ Each variable in the model was tested independently across a range of possible values to determine the impact of different assumptions on the cost results. Key assumptions were tested with the use of sensitivity analysis, in which the effects of simultaneously changing multiple variables were analyzed. 


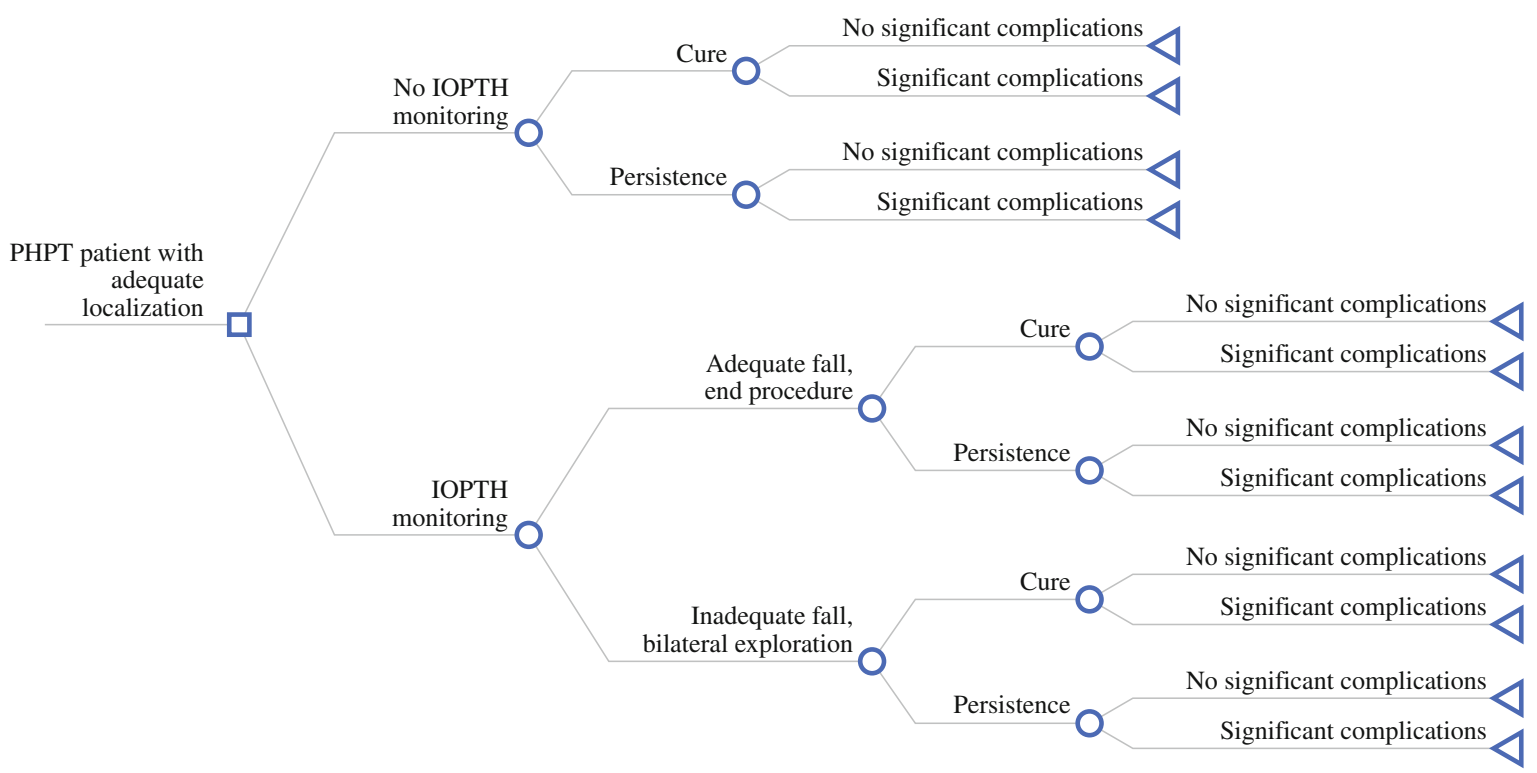

FIG. 1 Complete decision model for management of localized primary hyperparathyroidism with or without intraoperative parathyroid hormone monitoring

TABLE 1 Probabilities used in decision-tree analysis and supporting references

IOPTH intraoperative parathyroid hormone, $P H P T$ primary hyperparathyroidism

\begin{tabular}{lll}
\hline Description & $\begin{array}{l}\text { Probability } \\
\text { used }\end{array}$ & References \\
\hline $\begin{array}{l}\text { Probability of clinically important complication } \\
\quad \text { after multigland exploration }\end{array}$ & 0.02 & $10,11,32,33$ \\
$\begin{array}{l}\text { Probability of clinically important complication } \\
\text { after single-gland exploration }\end{array}$ & 0.01 & 10,11 \\
$\begin{array}{l}\text { Probability of cure with IOPTH monitoring } \\
\text { Probability of cure without IOPTH monitoring }\end{array}$ & 0.988 & $8,11,15,17,20,25,27,29,34-37$ \\
Probability that IOPTH decreases in localized PHPT & 0.963 & $7-9,15,18,19,21,25,28,36-38$ \\
\hline
\end{tabular}

TABLE 2 Costs by Medicare diagnosis-related group used in decision-tree analysis

\begin{tabular}{lr}
\hline Description & Cost (\$) \\
\hline Cost of clinically important complications & 5166.67 \\
Cost of IOPTH & 266.24 \\
Cost of multigland surgery & 4433.33 \\
Cost of reoperation & 5710.89 \\
Cost of single-gland parathyroidectomy & 3733.33
\end{tabular}

IOPTH intraoperative parathyroid hormone

\section{RESULTS}

In our model, the base case assumption was that IOPTH improves the operative success rate from 96.3 to $98.8 \%$. At baseline conditions, the IOPTH strategy increased the treatment cost by $3.8 \%$. IOPTH was cost saving when assay-related costs were less than $\$ 110$ per case (Fig. 2), or when the cost of reoperation exceeded $\$ 12,000$ (Fig. 3).
TABLE 3 Estimated operating room time by type of procedure performed

\begin{tabular}{lll}
\hline $\begin{array}{l}\text { Time } \\
(\mathrm{min})\end{array}$ & $\begin{array}{l}\text { Cost } \\
(\$)^{\mathrm{a}}\end{array}$ & Procedure \\
\hline 45 & 160.02 & $\begin{array}{l}\text { MIP without IOPTH } \\
\text { MIP with IOPTH, adequate decrease, end } \\
\text { procedure }\end{array}$ \\
15 & $\begin{array}{c}\text { MIP with IOPTH, inadequate decrease, convert } \\
\text { to BNE }\end{array}$
\end{tabular}

$M I P$ minimally invasive parathyroidectomy, IOPTH intraoperative parathyroid hormone monitoring, $B N E$ bilateral neck exploration

${ }^{\text {a }}$ Cost $=[6+($ time in minutes/15) $* 17.78]$

b IOPTH decrease of $\geq 50 \%$ from the highest of either preincision or preexcision level at $10 \mathrm{~min}$ after gland excision

The probability of failure to cure PHPT by single-gland parathyroidectomy without IOPTH monitoring is a surrogate for the rate of unrecognized MGD in patients with well-localized disease. If the probability of cure without IOPTH monitoring is $>94 \%$, under base case IOPTH and 


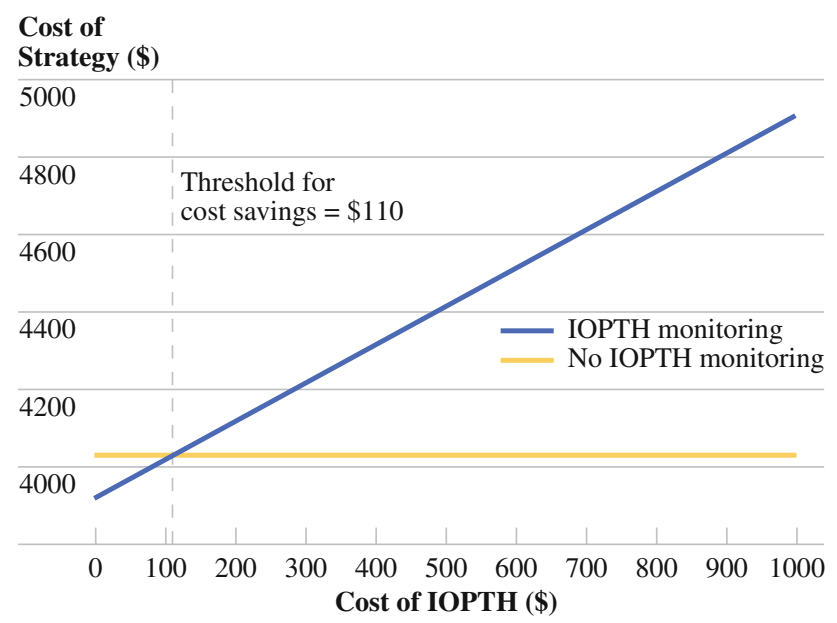

FIG. 2 One-way sensitivity analysis of cost of intraoperative parathyroid hormone (IOPTH). IOPTH monitoring becomes cost saving when test-related costs fall below $\$ 110$. This value considers both the cost of the test and the cost of operating room time spent waiting for results

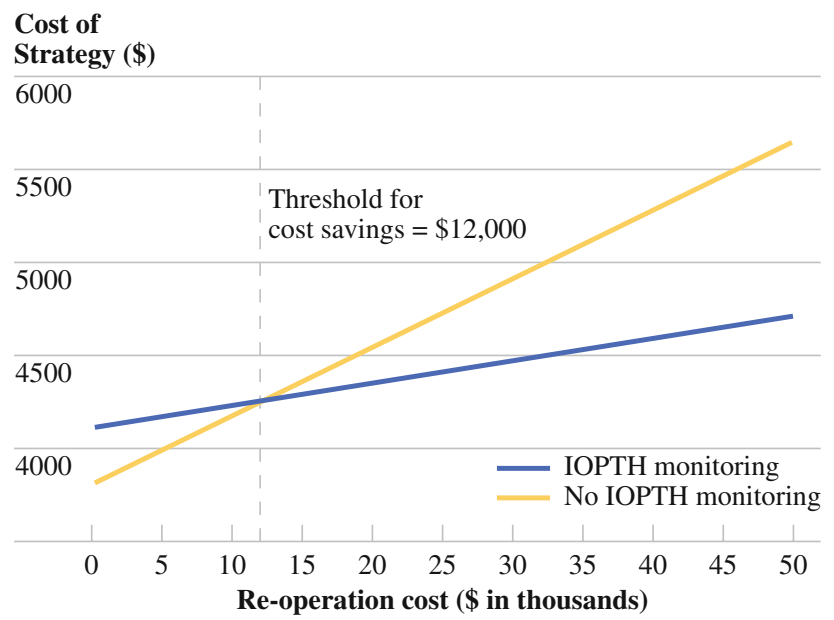

FIG. 3 One-way sensitivity analysis of reoperation cost. Intraoperative parathyroid hormone (IOPTH) monitoring becomes cost-saving when the reoperation cost increases above $\$ 12,000$. The difference in the slopes of the two lines reflects the marginal improvement in success rate of initial surgery associated with IOPTH monitoring in the model

reoperation costs, the use of IOPTH will increase treatment costs (Fig. 4). If the probability of cure without IOPTH monitoring is $<94 \%$, the use of IOPTH will reduce treatment costs. Two-way sensitivity analyses also yielded threshold values for cost savings of IOPTH on the basis of test-related costs and cost of reoperation, according to the probability of cure without IOPTH monitoring (Figs. 5, 6). As the probability of cure without IOPTH decreases to $<90 \%$, IOPTH monitoring becomes the dominant strategy in both analyses. Conversely, as the probability of cure without IOPTH exceeds $98 \%$, it is never cost saving to use

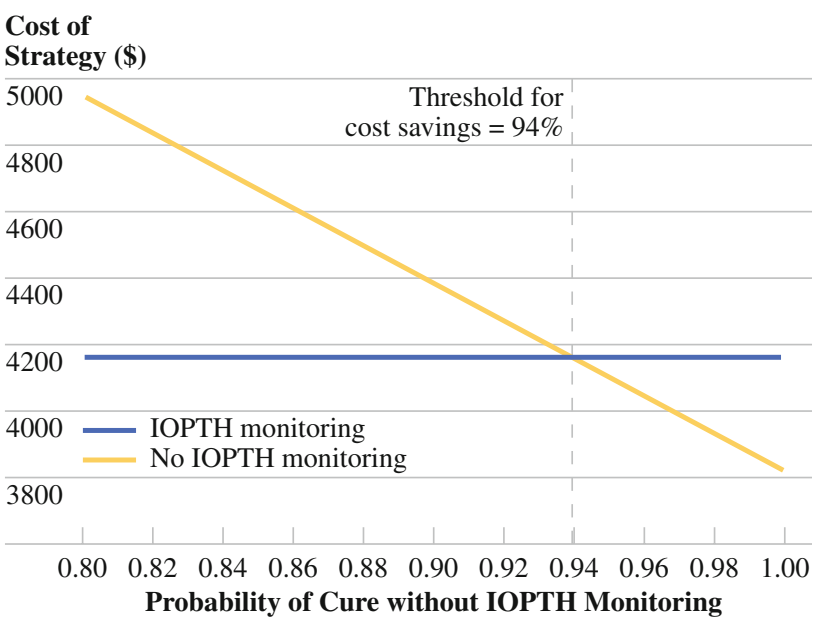

FIG. 4 One-way sensitivity analysis of probability of cure without intraoperative parathyroid hormone (IOPTH) monitoring. IOPTH monitoring becomes cost saving when the probability of cure without IOPTH monitoring decreases below 94\%. In the model, this probability relates inversely with the prevalence of undetected multiple gland disease among patients with positive localization studies

IOPTH monitoring, even when test-related costs are as low as $\$ 25$ and the cost of reoperation is $\$ 50,000$.

We also performed sensitivity analysis on the performance characteristics of IOPTH monitoring. In our model, the rates of false-positive and false-negative IOPTH results are represented by the probability that IOPTH decreases when a single localized gland is removed. Raising the probability that IOPTH decreases to $100 \%$ simultaneously optimizes the positive predictive value of IOPTH and eliminates false-negative results that would prompt unnecessary continued exploration. In two-way sensitivity analysis, the use of an ideally performing IOPTH test is cost saving when the probability of cure without IOPTH monitoring decreases below 94\% (Fig. 7). Less-than-perfect IOPTH test performance requires a lower probability of cure without IOPTH monitoring in order for IOPTH to become cost saving.

\section{DISCUSSION}

The true cost of IOPTH monitoring must be assessed on a hospital population basis as represented by the following equation ${ }^{6}$ :

Population cost of IOPTH monitoring

$=$ Population cost of IOPTH monitoring in every patient

+ Cost of unnecessary continued explorations

- Cost savings from avoided failures

In other words, although a relatively small fraction of patients with localized disease truly benefit from the use of 


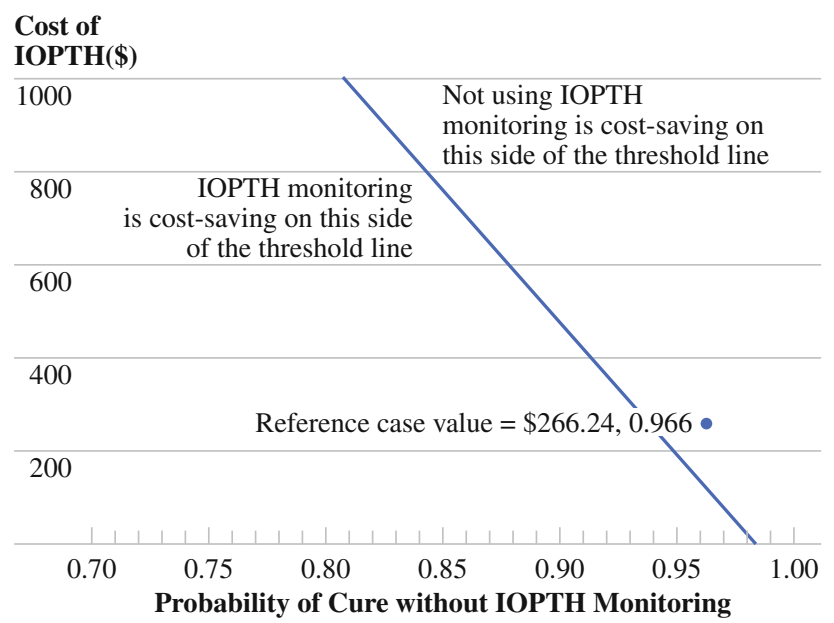

FIG. 5 Two-way sensitivity analysis of cost of intraoperative parathyroid hormone (IOPTH) and probability of cure without IOPTH monitoring. The reference case values, derived from Medicare diagnosis-related groups and literature review, are shown. When all other assumptions in the model are held constant, this analysis permits cost assessment of IOPTH monitoring among institutions with different published rates of undetected multiple gland disease (MGD). When the MGD rate is 22\% (Cleveland (16)), IOPTH monitoring is always cost saving, irrespective of test-related costs. When the MGD rate is $10 \%$ (Madison (8)), IOPTH monitoring becomes cost saving as test-related costs fall below $\$ 440$. When the MGD rate is 2\% (Sydney (5)), IOPTH monitoring is never cost saving

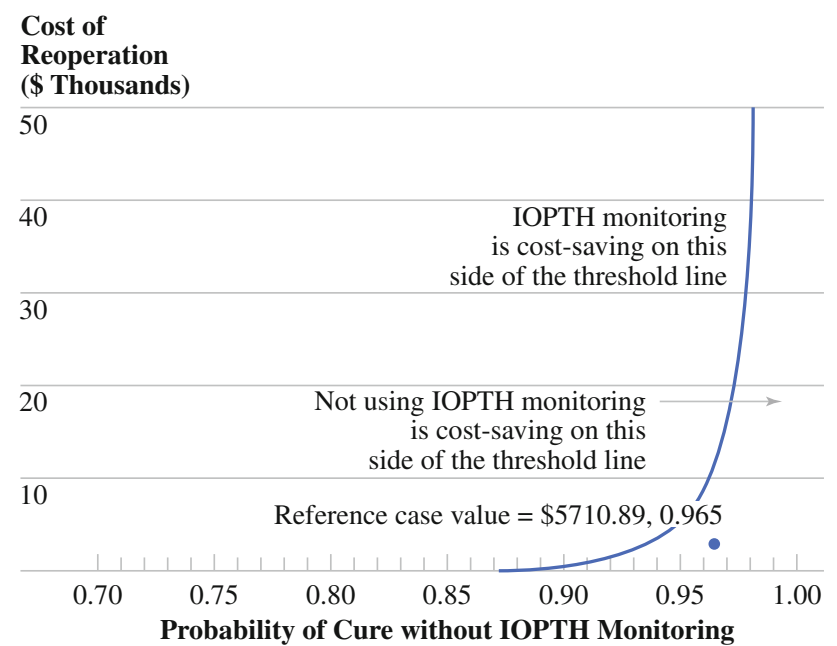

FIG. 6 Two-way sensitivity analysis of cost of reoperation and probability of cure without intraoperative parathyroid hormone (IOPTH) monitoring. The $y$-axis is inclusive of an approximately ninefold error in reoperation cost. The reference case values are shown

IOPTH, the cost of IOPTH monitoring is borne broadly. Institutions that routinely use IOPTH monitoring might fall into three general categories regarding the justification for this added cost: (1) centers that have high rates of unrecognized MGD among patients with positive localization, either as a result of a high overall prevalence of MGD or

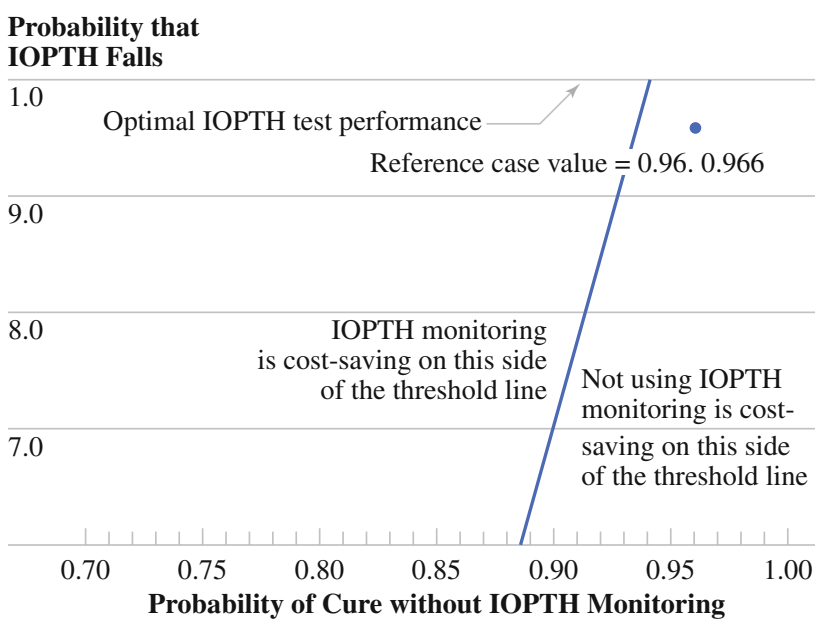

FIG. 7 Two-way sensitivity analysis of probability that intraoperative parathyroid hormone (IOPTH) decreases and probability of cure without IOPTH monitoring. Raising the probability that IOPTH decreases to $100 \%$ optimizes test performance. The reference case values are shown

relatively low accuracy of preoperative localization studies; (2) centers that leverage IOPTH monitoring to begin all cases as limited explorations, irrespective of imaging results; and (3) centers that aim to cure $100 \%$ of patients, regardless of cost. ${ }^{8,11,15-19}$ Detractors primarily focus on the limited "added value" of IOPTH monitoring, arguing that a $1 \%$ improvement in success rates with IOPTH monitoring is not merited by the high cost of the test, as well as the false-negative rate of $1.2-9.8 \%$ that leads to unnecessary continued explorations. ${ }^{5,10,20,21}$

It is increasingly evident that the contribution of IOPTH monitoring varies inversely with the accuracy of localization studies. Even the strongest advocates of IOPTH monitoring admit to its marginal benefit in patients with definitive preoperative localization. ${ }^{22}$ The ostensible purpose of IOPTH monitoring in localized PHPT is to unmask unrecognized MGD; however, the ability of the test to successfully serve this function is not certain. Perversely, the accuracy of IOPTH is reduced in the presence of MGD, prompting some experts to comment that "the test works best when it is needed least." 23,24

Our literature review of predominantly single-institution studies revealed wide variation in several critical factors that were likely to influence the surgeon's decision to use IOPTH. The most striking of these was the prevalence of unrecognized MGD in patients with positively localized disease, which ranged from 1.6 to $22 \% .^{5,7,10,15-19,25-29}$ This variation prompted us to surmise that divided opinions regarding the value of IOPTH monitoring rest not on dogma but rather arise as rational adaptations to disparate institution-specific factors. Our analyses are thus inclusive of wide ranges in MGD rate, IOPTH cost, and reoperation cost. 
True costs of IOPTH vary and are difficult to quantify. Costs associated with IOPTH include cost of the assay, the machine, and a dedicated technician, with three to five assays per case. Most assays take $8-16$ min to deliver a result. Because the 10-min postexcision parathyroid hormone level is the one most typically relied on, total waiting time in the OR can approach $30 \mathrm{~min}$. From the third-party payer cost perspective used in our model, the marginal cost of $1 \mathrm{~min}$ of OR time is $\$ 1.19$, a value that we based on the following Medicare formula, which includes only the cost of anesthesia: Cost $=[6+($ time in minutes $/ 15) * 17.78]$. The IOPTH cost used in the model (\$266.24) considers this plus an expected payment of $\$ 56.76$ for each of four assays used per case. In contrast to these figures, the cost of OR time in a recent surgical workflow study was $\$ 15.05$ per minute. ${ }^{30}$ Incorporation of such real-world time costs would work considerably against IOPTH monitoring in decision analysis.

Because our threshold value for IOPTH cost was $\$ 110$, well under \$266.24, IOPTH monitoring did not reduce treatment costs under base case assumptions. However, the IOPTH cost only comprised a small fraction of the total cost of care per patient, ranging from a base of $4 \%$ to a maximum of $20 \%$ increase in cost when IOPTH cost was raised to $\$ 1000$.

Examination of Figs. 5, 6 and 7 reveals conditions when IOPTH monitoring is the cost-saving strategy. However, in most cited reports from expert centers, the probability of cure without IOPTH monitoring is $95 \%$ or more among localized cases, confining these institutions to the far righthand side of the two-way sensitivity analyses. Along the 95\% vertical, not using IOPTH is cost saving when the remaining assumptions are held constant.

We found that IOPTH monitoring became cost saving when the cost of reoperation exceeded $\$ 12,000$, which is more than three times the cost of initial MIP (\$3733). Although the cost of reoperation is certainly higher than that of the initial operation in published reports, the magnitude of the difference is well under threefold, in keeping with our own experience. ${ }^{31}$ This suggests that a strategy permissive of a small number of failed initial operations, rather than one aiming for an initial cure rate of $100 \%$, would be most cost conscious.

Several recent publications have described the application of second-tier localization studies in sestamibinegative patients, most notably parathyroid-protocol computed tomographic scans. Our analyses suggest that adding such an examination would only be cost saving if the probability of cure without computed tomography were unacceptably low. In practice, this scenario would likely occur in institutions where the performance of sestamibi scanning lay below the median found in our literature review.
Because our study did not include quality of life indicators, we are unable to comment on the cost-effectiveness of the strategies shown. Our analyses only indicate circumstances when IOPTH monitoring can be expected to negatively or positively affect the cost of care. Moreover, our study modeled cases with positive localization and findings are not generalizable to the overall population of patients with PHPT. We conclude that in the management of localized PHPT, the cost impact of IOPTH monitoring depends on institution-specific factors, most importantly the prevalence of unrecognized MGD. The marginally improved cure rate achieved with IOPTH monitoring was offset by an increased cost of care per patient.

OPEN ACCESS This article is distributed under the terms of the Creative Commons Attribution Noncommercial License which permits any noncommercial use, distribution, and reproduction in any medium, provided the original author(s) and source are credited.

\section{REFERENCES}

1. Sidhu S, Neill AK, Russell CF. Long-term outcome of unilateral parathyroid exploration for primary hyperparathyroidism due to presumed solitary adenoma. World J Surg. 2003;27:339-42.

2. Westerdahl J, Bergenfelz A. Unilateral versus bilateral neck exploration for primary hyperparathyroidism: five-year follow-up of a randomized controlled trial. Ann Surg. 2007;246:976-80.

3. Sackett WR, Barraclough B, Reeve TS, Delbridge LW. Worldwide trends in the surgical treatment of primary hyperparathyroidism in the era of minimally invasive parathyroidectomy. Arch Surg. 2002;137:1055-9.

4. Carling T, Udelsman R. Focused approach to parathyroidectomy. World J Surg. 2008;32:1512-7.

5. Stalberg P, Sidhu S, Sywak M, et al. Intraoperative parathyroid hormone measurement during minimally invasive parathyroidectomy: does it "value-add" to decision-making? J Am Coll Surg. 2006;203:1-6.

6. Agarwal G, Barakate MS, Robinson B, Wilkinson M, Barraclough B, Reeve TS, et al. Intraoperative quick parathyroid hormone versus same-day parathyroid hormone testing for minimally invasive parathyroiectomy: a cost-effectiveness study. Surgery. 2001;130:963-70.

7. Arici C, Cheah WK, Ituarte PHG, Morita E, Lynch EC, Siperstein $\mathrm{AE}$, et al. Can localization studies be used to direct focused parathyroid operations? Surgery. 2001;129:720-9.

8. Chen H, Pruhs Z, Starling JR, Mack E. Intraoperative parathyroid hormone testing improves cure rates in patients undergoing minimally invasive parathyroidectomy. Surgery. 2005;138:58390.

9. Mihai R, Palazzo FF, Gleeson FV, Sadler GP. Minimally invasive parathyroidectomy without intraoperative parathyroid hormone monitoring in patients with primary hyperparathyroidism. $\mathrm{Br} \mathrm{J}$ Surg. 2007;94:42-7.

10. Pang T, Stalberg P, Sidhu S, Sywak M, Wilkinson M, Reeve TS, et al. Minimally invasive parathyroidectomy using the lateral focused mini-incision technique without intraoperative parathyroid hormone monitoring. Br J Surg. 2007;94:315-9.

11. Udelsman R. Six hundred fifty-six consecutive explorations for primary hyperparathyroidism. Ann Surg. 2002;235:665-72.

12. Bilezikian JP, Potts JT Jr, Fuleihan Gel H, Kleerekoper M, Neer $\mathrm{R}$, Peacock M, et al. Summary statement from a workshop on 
asymptomatic primary hyperparathyroidism: perspective for the 21st century. J Clin Endocrinol Metab. 2002;87:5353-61.

13. Carniero D, Solorzano CC, Nader MC, Ramirez M, Irvin GL. Comparison of intraoperative $\mathrm{PTH}$ assay (QPTH) criteria in guiding parathyroidectomy: which criterion is the most accurate? Surgery. 2003;134:973-81.

14. Gold MR. Cost-effectiveness in health care and medicine. New York: Oxford University Press, 1996.

15. Siperstein A, Berber E, Mackey R, et al. Prospective evaluation of sestamibi scan, ultrasonography, and rapid PTH to predict the success of limited exploration for sporadic primary hyperparathyroidism. Surgery. 2004;136:872-80.

16. Sharma J, Milas M, Berber E, Mazzaglia P, Siperstein A, Weber CJ. Value of intraoperative parathyroid hormone monitoring. Ann Surg Oncol. 2007;15:493-8.

17. Irvin G III, Solorzano CC, Carneiro DM. Quick intraoperative parathyroid hormone assay: surgical adjunct to allow limited parathyroidectomy, improve success rate, and predict outcome. World J Surg. 2004;28:1287-92.

18. Solorzano CC, Carneiro-Pla DM, Irvin GL III. Surgeon-performed ultrasonography as the initial and only localizing study in sporadic primary hyperparathyroidism. $J$ Am Coll Surg. 2006;202:18-24.

19. Solorzano C, Lee TM, Ramirez MC, Carneiro DM, Irvin GL III. Surgeon-performed ultrasound improves localization of abnormal parathyroid glands. Am Surg. 2005;71:557-63.

20. Miura D, Wada N, Arici C, Morita E, Duh QY, Clark OH. Does intraoperative quick parathyroid hormone assay improve the results of parathyroidectomy? World J Surg. 2002;26:926-30.

21. Jacobson S, van Heerden JA, Farley DA, Grant, CS, Thompson, GB, Mullan, BP, et al. Focused cervical exploration for primary hyperparathyroidism without intraoperative parathyroid hormone monitoring or use of the gamma probe. World J Surg. 2004;28:1127-31.

22. Lew JI, Solorzano CC, Montano RE, Carneiro-Pla DM, Irvin GL III. Role of intraoperative parathormone monitoring during parathyroidectomy in patients with discordant localization studies. Surgery. 2008;144:299-306.

23. Gauger PG, Agarwal G, England BG, Delbridge LW, Matz KA, Wilkinson $\mathrm{M}$, et al. Intraoperative parathyroid hormone monitoring fails to detect double parathyroid adenomas: a 2-institution experience. Surgery. 2001;130:1005-10.

24. Duh Q. What's new in general surgery: endocrine surgery. $J$ Am Coll Surg. 2005;201:746-53.

25. Chiu B, Sturgeon C, Angelos P. What is the link between nonlocalizing sestamibi scans, multigland disease, and persistent hypercalcemia? A study of 401 consecutive patients undergoing parathyroidectomy. Surgery. 2006;140:418-22.
26. Irvin G III, Carneiro DM, Solorzano CC. Progress in the operative management of sporadic primary hyperthyroidism over 34 years. Ann Surg. 2004;239:704-11.

27. Quiros R, Alioto J, Wilhelm SM, Ali A, Prinz RA. An algorithm to maximize the use of minimally invasive parathyroidectomy. Arch Surg. 2004;139:501-7.

28. Soon P, Delbridge LW, Sywak MS, Barraclough BM, Edhouse P, Sidhu SB. Surgeon performed ultrasound facilitates minimally invasive parathyroidectomy by the focused lateral mini-incision approach. World J Surg. 2008;32:766-71.

29. Yip L, Pryma DA, Yim JH, Virji MA, Carty SE, Ogilvie JB. Can a lightbulb sestamibi SPECT accurately predict single-gland disease in sporadic primary hyperparathyroidism? World J Surg. 2008;32:784-92.

30. Stahl J, Sandberg WS, Daily B, Wiklund R, Egan MT, Goldman JM. Reorganizing patient care and workflow in the operating room: a cost-effectiveness study. Surgery. 2006;139:717-28.

31. Doherty GM, Weber B, Norton JA. Cost of unsuccessful surgery for primary hyperparathyroidism. Surgery. 1994;116:954-7.

32. Allendorf J, DiGorgi M, Spanknebel K, Inabnet W, Chabot J, Logerfo P. 1112 consecutive bilateral neck explorations for primary hyperparathyroidism. World J Surg. 2007;31:2075-80.

33. Sosa J, Powe NR, Levine MA, Udelsman R, Zeiger MA. Thresholds for surgery and surgical outcomes for patients with primary hyperparathyroidism: a national survey of endocrine surgeons. J Clin Endocrinol Metab. 1996;83:2658-65.

34. Grant C, Thompson G, Farley D, van Heerden J. Primary hyperparathyroidism surgical management since the introduction of minimally invasive parathyroidectomy. Arch Surg. 2005; 140:472-9.

35. Genc H, Morita E, Perrier ND, Miura D, Ituarte P, Duh Q-Y, et al. Differing histologic findings after bilateral and focused parathyroidectomy. J Am Coll Surg. 2003;196:535-40.

36. Beyer T, Solorzano CC, Starr F, Nilubol N, Prinz RA. Parathyroidectomy outcomes according to operative approach. Am J Surg. 2007;193:368-73.

37. Rodgers S, Hunter GJ, Hamberg LM, Schellingerhout D, Doherty $\mathrm{DB}$, Ayers GD, et al. Improved preoperative planning for directed parathyroidectomy with 4-dimensional computed tomography. Surgery. 2006;140:932-40.

38. Kebebew E, Hwang J, Reiff E, Duh QY, Clark OH. Predictors of single-gland vs multigland parathyroid disease in primary hyperparathyroidism. Arch Surg. 2006;141:777-82.

39. Chen H, Mack E, Starling JR. A comprehensive evaluation of perioperative adjuncts during minimally invasive parathyroidectomy: which is most reliable? Ann Surg. 2005;242:375-83. 